\title{
Efficient synthesis of a 3,6-branched mannose hepta- and octasaccharide
}

\author{
Jianjun Zhang, Zuchao Ma, Fanzuo Kong* \\ Research Center for Eco-Environmental Sciences, Academia Sinica, Chinese Academy of Sciences, P.O. Box 2871, Beijing 100085, PR China
}

Received 29 May 2003; accepted 14 July 2003

\begin{abstract}
$\alpha$-D-Manp - $(1 \rightarrow 3)-[\alpha-\mathrm{D}-\operatorname{Man} p-(1 \rightarrow 2)-\alpha-\mathrm{D}-\operatorname{Man} p-(1 \rightarrow 6)]-\alpha-\mathrm{D}-\operatorname{Man} p-(1 \rightarrow 3)-[\alpha-\mathrm{D}-\mathrm{Man} p-(1 \rightarrow 2)-\alpha-\mathrm{D}-\mathrm{Man} p-(1 \rightarrow 6)]-\mathrm{D}-\mathrm{Man} p$ and $\alpha$-D-Man $p-(1 \rightarrow 2)-\alpha-D-M a n p-(1 \rightarrow 3)-[\alpha-D-M a n p-(1 \rightarrow 2)-\alpha-D-M a n p-(1 \rightarrow 6)]-\alpha-D-M a n p-(1 \rightarrow 3)-[\alpha-D-M a n p-(1 \rightarrow 2)-\alpha-D-M a n p-(1 \rightarrow$ $6)$ ]-D-Man $p$, were synthesized as their methyl glycosides in a regio- and stereoselective way.

(C) 2003 Elsevier Ltd. All rights reserved.

Keywords: Oligosaccharide; Mannose; Synthesis
\end{abstract}

\section{Introduction}

Asparagine-linked glycoprotein oligosaccharides play a vital role in fundamental biological processes such as cell differentiation, malignant transformation, viral, bacterial and parasitic infections and protein transportations. ${ }^{1}$ They are usually divided into three major subgroups, i.e., high-mannose type, complex type and hybrid type, and each of them may consist of diverse structures in living cells. ${ }^{2}$ A 3,6-branched mannose nonasaccharide of the $\mathrm{N}$-glycan is expressed on the HIV gp 120 glycoprotein, ${ }^{3}$ whereas a 3,6-branched mannose octasaccharide of the $\mathrm{N}$-glycan exists in the adhesion domain of human CD2 (Scheme 1). ${ }^{4}$

As an ongoing project on the research on structurebioactivity relationship among oligosaccharides, we have synthesized (Scheme 2) a variety of naturally occurring mannose oligosaccharides, such as $(1 \rightarrow 2)$ branched $(1 \rightarrow 6)$-linked mannans ${ }^{5}$ and 3,6-branched mannans, ${ }^{6}$ and also a series of artificially designed oligosaccharides. ${ }^{7}$ It was interesting to find that some synthetic glucose oligosaccharides showed excellent bioactivity compared to the natural ones. ${ }^{7}$ In continuation of our studies on oligosaccharides, we present

\footnotetext{
* Corresponding author. Tel.: +86-10-62936613; fax: +8610-62923563.

E-mail address: fzkong@mail.rcees.ac.cn (F. Kong).
}

herein syntheses of the mannose hepta- and octasaccharide analogues of the N-glycan mannans.

\section{Results and discussion}

Our previous communication ${ }^{8}$ described an efficient synthesis of the hexasaccharide repeating unit of the exopolysaccharide (GXM) from Cryptococcus neoformans serovar A with 4,6- $O$-isopropylidenated mannose derivatives as the key synthons. We found that the 4,6$O$-isopropylidenated mannose derivatives were also appropriate intermediates in the present research for construction of the required mannose hepta- and octasaccharides. As outlined in Scheme 3, 1,2,3-tri- $O$ acetyl-4,6- $O$-isopropylidene-D-mannopyranose (1), obtained from selective 4,6- $O$-isopropylidenation of mannose $^{9}$ with 2-methoxypropene, followed by acetylation, was chosen as the starting material. Selective 1-Odeacetylation with ammonia in $\mathrm{THF}-\mathrm{MeOH}$, followed by trichloroacetimidation ${ }^{10}$ with trichloroacetonitrile in the presence of potassium carbonate, gave the donor 2,3-di- $O$-acetyl-4,6- $O$-isopropylidene- $\alpha$-D-mannopyranosyl trichloroacetimidate (2). Condensation of 2 with the acceptor, methyl 4,6- $O$-isopropylidene- $\alpha$-D-mannopyranoside (3), selectively afforded the $(1 \rightarrow 3)$-linked disaccharide $4(74 \%)$. The regioselectivity of the coupling was confirmed by acetylation of $\mathbf{4}$ to give $\mathbf{5}$, and 

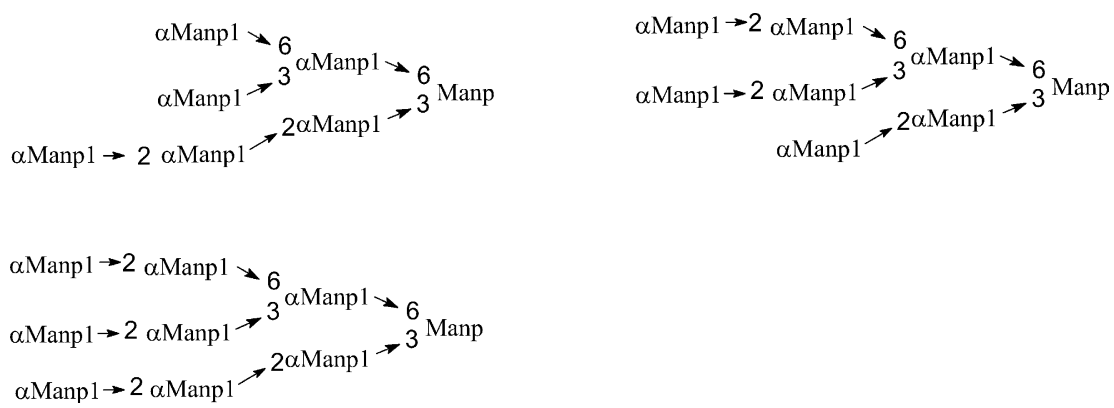

Scheme 1. Examples of naturally occurring N-glycans Man7, Man8, and Man9.

the ${ }^{1} \mathrm{H}$ NMR spectrum of 5 showed a newly emerged downfield doublet of doublets at $\delta 5.34 \mathrm{ppm}$ with $J_{1,2}=$ 1.5 and $J_{2,3}=3.0 \mathrm{~Hz}$ for $\mathrm{H}-2$ compared to that of 4 . Deacetylation of $\mathbf{4}$ or $\mathbf{5}$ in a solution of ammonia in methanol furnished the disaccharide triol acceptor $\mathbf{6}$ in high yield. Again, 3- $O$-selective glycosylation of 6 with the donor, 2- $O$-acetyl-3,4,6-tri- $O$-benzoyl- $\alpha$-D-mannopyranosyl- $(1 \rightarrow 2)-3,4,6$-tri- $O$-benzoyl- $\alpha$-D-mannopyranosyl trichloroacetimidate ${ }^{11}(7)$, yielded $(1 \rightarrow 3)$-linked tetrasaccharide $8(71 \%)$. The 3-O-glycosylation was identified by acetylation to give 9 , and the ${ }^{1} \mathrm{H}$ NMR spectrum of 9 showed three characteristic signals at $\delta$ $5.66 \mathrm{ppm}$ with $J_{1,2}=0.7$ and $J_{2,3}=2.7 \mathrm{~Hz}, 5.41 \mathrm{ppm}$ with $J_{1,2}=0.8$ and $J_{2,3}=3.0 \mathrm{~Hz}$, and $5.11 \mathrm{ppm}$ with $J_{1,2}=1.0$ and $J_{2,3}=3.0 \mathrm{~Hz}$ for $\mathrm{H}^{\prime \prime \prime}-2, \mathrm{H}^{\prime}-2$, and $\mathrm{H}-2$, respectively. Removal of the $O$-isopropylidene groups was smoothly carried out with $90 \%$ TFA giving the tetrasaccharide acceptor $\mathbf{1 0}(83 \%)$. Selective 6- $O$-glycosylation $^{12}$ of $\mathbf{1 0}$ with the disaccharide donor $\mathbf{7}$ afforded octasaccharide 11 (61\%), and subsequent acetylation gave 12. The ${ }^{1} \mathrm{H}$ NMR spectrum of 12 showed eightproton signals for $\mathrm{H}-4$ at the region of $\delta$ 5.2-6.2, indicating the 6-O-selective glycosylation. Deacylation of $\mathbf{1 2}$ in a saturated solution of ammonia in methanol furnished the target octasaccharide. The heptasaccharide 20 was obtained in a similar way except that, instead of the disaccharide donor 7, 2-O-acetyl-3,4,6-tri- $O$ benzoyl- $\alpha$-D-mannopyranosyl trichloroacetimidate (14) was used to couple $\mathbf{6}$. The rest of the steps were exactly the same as those used for the preparation of $\mathbf{1 3}$ from $\mathbf{8}$. Bioactivity tests for $\mathbf{1 3}$ and $\mathbf{2 0}$ are in progress.

In summary, 3,6-branched mannose hepta- and octasaccharide analogues of the $\mathrm{N}$-glycan mannans were synthesized in a highly regio- and steroselective way with a quite simple procedure. Large-scale preparations should be possible with this method.

\section{Experimental}

\subsection{General methods}

Melting points were determined with a 'Mel-Temp' apparatus. Optical rotations were determined with a Perkin-Elmer model 241-MC automatic polarimeter for solutions in a 1-dm, jacketed cell. ${ }^{1} \mathrm{H},{ }^{13} \mathrm{C}$, and $2 \mathrm{D}$ NMR spectra were recorded with Varian XL-400 spectrometers, for solutions in $\mathrm{CDCl}_{3}$ or in $\mathrm{D}_{2} \mathrm{O}$ as indicated. Individual resonances could not identified with the specific sugar residues. Chemical shifts are expressed in ppm downfield from the $\mathrm{Me}_{4} \mathrm{Si}$ absorption. Mass spectra were recorded with a VG PLATFORM mass spectrometer using the ESI mode. Thin-layer chromatography (TLC) was performed on silica gel HF with detection by charring with $30 \%(\mathrm{v} / \mathrm{v})$ sulfuric acid in methanol or by UV detection. Column chromatography was conducted by elution of a column $(8 \times 100,16 \times$ $240,18 \times 300,35 \times 400 \mathrm{~mm})$ of silica gel $(100-200$ mesh) with EtOAc-petroleum ether (bp $60-90^{\circ} \mathrm{C}$ ) as the eluent. Analytical LC was performed with a Gilson HPLC consisting of a pump (model 306), stainless steel column packed with silica gel (Spherisorb $\mathrm{SiO}_{2}, 10 \times 300$ or $4.6 \times 250 \mathrm{~mm})$, differential refractometer (132-RI Detector), UV-Vis detector (model 118). EtOAc-petroleum ether (bp $60-90{ }^{\circ} \mathrm{C}$ ) was used as the eluent at a
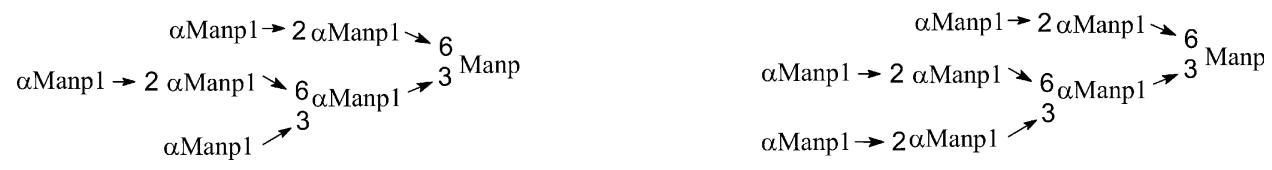

Scheme 2. Synthetic mannose hepta- and octasaccharides. 

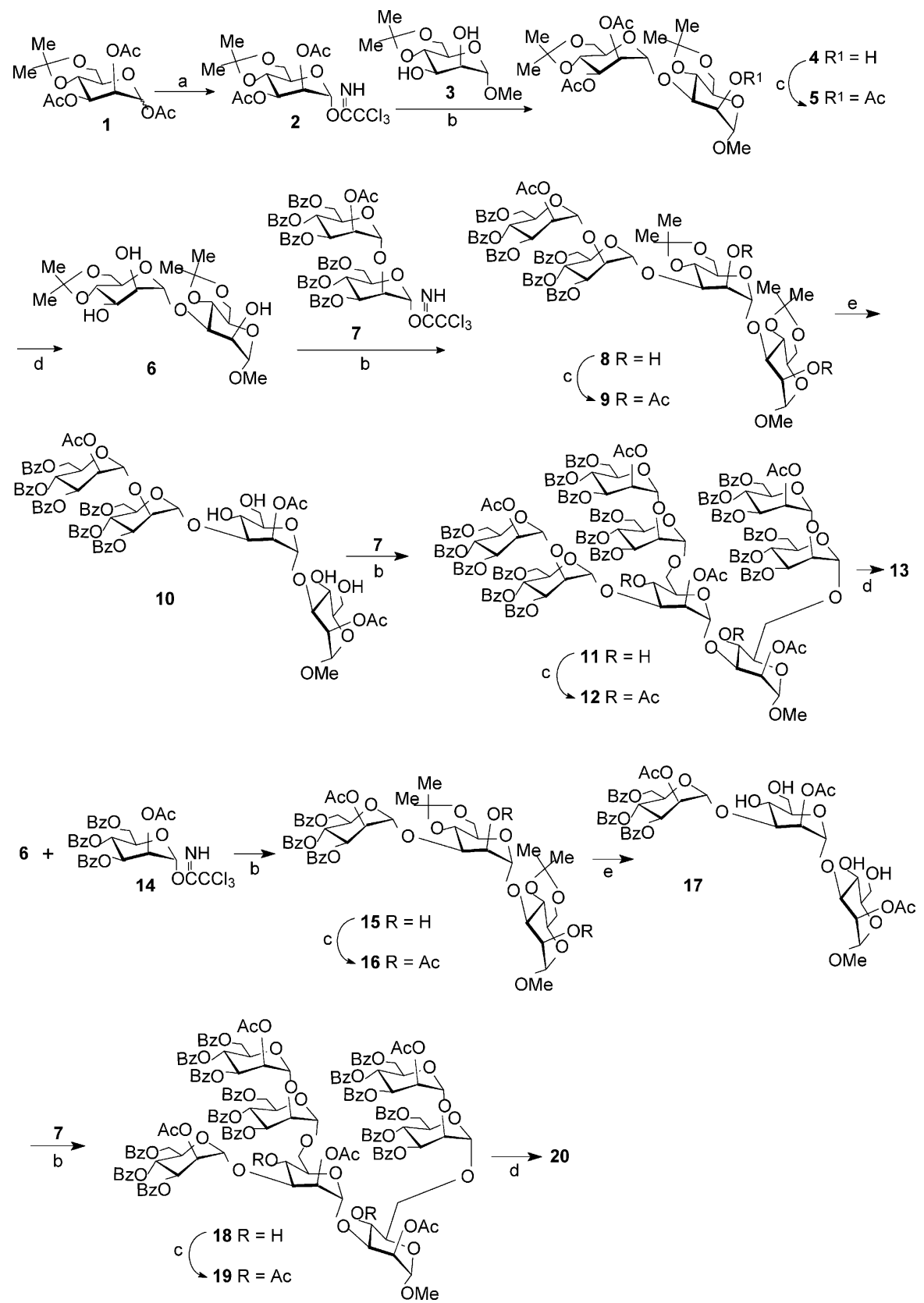

Scheme 3. Reagents and conditions: (a) (i) THF- $\mathrm{CH}_{3} \mathrm{OH}, 1.5 \mathrm{~N} \mathrm{NH}$, rt, $2-3 \mathrm{~h}$; (ii) $\mathrm{CH}_{2} \mathrm{Cl}_{2}, \mathrm{CCl}_{3} \mathrm{CN}$ (2.0 equiv), $\mathrm{K}_{2} \mathrm{CO}_{3}(2.0$ equiv), rt, $12 \mathrm{~h}, 71 \%$. (b) TMSOTf ( $0.05-0.10$ equiv), $4 \AA \mathrm{MS}, \mathrm{CH}_{2} \mathrm{Cl}_{2},-20^{\circ} \mathrm{C}, 2-4 \mathrm{~h}$ ( $74 \%$ for $\mathbf{4}, 71 \%$ for $\mathbf{8}, 61 \%$ for $\mathbf{1 1}, 67 \%$ for $\mathbf{1 5}, 75 \%$ for 18). (c) $\mathrm{Ac}_{2} \mathrm{O}-$-Pyridine, $81-93 \%$. (d) $\mathrm{CH}_{3} \mathrm{OH}$ saturated with ammonia, rt, $12-36 \mathrm{~h}, 73-96 \%$. (e) $90 \% \mathrm{TFA}, \mathrm{rt}, 2 \mathrm{~h}, 83 \%$ for $10,80 \%$ for 17 .

flow rate of $1-4 \mathrm{~mL} / \mathrm{min}$. Solutions were concentrated at a temperature $<60{ }^{\circ} \mathrm{C}$ under diminished pressure.

\subsection{2,3-Di- $O$-acetyl-4,6- $O$-isopropylidene- $\alpha$-D- mannopyranosyl trichloroacetimidate (2)}

Compound 1 ( $6.90 \mathrm{~g}, 20.0 \mathrm{mmol})$ was dissolved in $1 \mathrm{M}$ solution of ammonia-methanol $(100 \mathrm{~mL})$ and stirred for $4 \mathrm{~h}$, at the end of which time TLC (3:1 petroleum
ether-EtOAc) indicated that the reaction was complete. The solution was concentrated and dried under high vacuum giving a white foamy solid. This foamy solid was dissolved in dry dichloromethane $(50 \mathrm{~mL})$, then trichloroacetonitrile $(6.3 \mathrm{~mL}, 30 \mathrm{mmol})$ and 1,8-diazabicyclo[5.4.0]undecene (DBU) $(0.50 \mathrm{~mL}, 4.04 \mathrm{mmol})$ was added subsequently. The reaction mixture was stirred under nitrogen for $3 \mathrm{~h}$ and then concentrated. The residue was purified by chromatography (4:1 
petroleum ether-EtOAc) to give $2(7.40 \mathrm{~g}, 82.2 \%)$ as a syrup: $[\alpha]_{\mathrm{D}}+42.0^{\circ}\left(c 1.0, \mathrm{CHCl}_{3}\right) ;{ }^{1} \mathrm{H}$ NMR $(400 \mathrm{MHz}$, $\left.\mathrm{CDCl}_{3}\right): \delta 8.72\left(\mathrm{~s}, 1 \mathrm{H}, \mathrm{CNHCCL}{ }_{3}\right), 6.17\left(\mathrm{~s}, 1 \mathrm{H}, J_{1,2} 1.6\right.$ $\mathrm{Hz}, \mathrm{H}-1), 5.50\left(\mathrm{dd}, 1 \mathrm{H}, J_{1,2} 1.6 \mathrm{~Hz}, J_{2,3} 3.4 \mathrm{~Hz}, \mathrm{H}-2\right)$, $5.30\left(\mathrm{dd}, 1 \mathrm{H}, J_{2,3} 3.4 \mathrm{~Hz}, J_{3,4} 10.3 \mathrm{~Hz}, \mathrm{H}-3\right), 4.11(\mathrm{dd}, 1$ $\left.\mathrm{H}, J_{3,4} 10.3 \mathrm{~Hz}, J_{4,5} 10.0 \mathrm{~Hz}, \mathrm{H}-4\right), 3.98-3.85(\mathrm{~m}, 3 \mathrm{H})$, $2.20\left(\mathrm{~s}, 3 \mathrm{H}, \mathrm{CH}_{3} \mathrm{CO}\right), 2.04\left(\mathrm{~s}, 3 \mathrm{H}, \mathrm{CH}_{3} \mathrm{CO}\right), 1.55(\mathrm{~s}, 3$ $\mathrm{H}$, isopropylidene), 1.42 (s, $3 \mathrm{H}$, isopropylidene). Anal. Calcd for $\mathrm{C}_{15} \mathrm{H}_{20} \mathrm{Cl}_{3} \mathrm{NO}_{8}$ : C, 40.12; H, 4.49. Found: $\mathrm{C}$, 40.40; H, 4.73.

\subsection{Methyl 2,3-di- $O$-acetyl-4,6- $O$-isopropylidene- $\alpha$-D- mannopyranosyl-( $1 \rightarrow 3)-4,6-O$-isopropylidene- $\alpha$-D- mannopyranoside (4)}

To a cooled solution $\left(-20{ }^{\circ} \mathrm{C}\right)$ of $3(1.17 \mathrm{~g}, 5.0 \mathrm{mmol})$ and $2(2.46 \mathrm{~g}, 5.5 \mathrm{mmol})$ in anhyd $\mathrm{CH}_{2} \mathrm{Cl}_{2}(50 \mathrm{~mL})$ was added TMSOTf $(18 \mu \mathrm{L}, 0.05 \mathrm{mmol})$. The mixture was stirred at this temperature for $2 \mathrm{~h}$, and then quenched with $\mathrm{Et}_{3} \mathrm{~N}$ (two drops). The solvents were evaporated in vacuo to give a residue, which was purified by silica gel column chromatography (2:1 petroleum ether-EtOAc) to give disaccharide $4(1.93 \mathrm{~g}, 74.2 \%)$ as a syrup: $[\alpha]_{\mathrm{D}}+$ 43.3 (c 1.0, $\mathrm{CHCl}_{3}$ ); ${ }^{1} \mathrm{H}$ NMR (400 $\mathrm{MHz}, \mathrm{CDCl}_{3}$ ): $\delta$ $5.36\left(\mathrm{dd}, 1 \mathrm{H}, J_{1,2} 1.0 \mathrm{~Hz}, J_{2,3} 3.2 \mathrm{~Hz}, \mathrm{H}-2^{\prime}\right), 5.21(\mathrm{dd}, 1$ $\left.\mathrm{H}, J_{2,3} 3.2 \mathrm{~Hz}, J_{3,4} 9.9 \mathrm{~Hz}, \mathrm{H}-3^{\prime}\right), 5.20\left(\mathrm{~d}, 1 \mathrm{H}, J_{1,2} 1.0\right.$ $\left.\mathrm{Hz}, \mathrm{H}-1^{\prime}\right), 4.73$ (d, $\left.1 \mathrm{H}, J_{1,2} 1.1 \mathrm{~Hz}, \mathrm{H}-1\right), 4.14(\mathrm{dd}, 1 \mathrm{H}$, $\left.J_{3,4}=J_{4,5}=9.9 \mathrm{~Hz}, \mathrm{H}-4\right), 4.06-4.02(\mathrm{~m}, 2 \mathrm{H}), 3.95(\mathrm{dd}, 1$ $\left.\mathrm{H}, J_{2,3} 3.3 \mathrm{~Hz}, J_{3,4} 10.0 \mathrm{~Hz}, \mathrm{H}-3\right), 3.87-3.76(\mathrm{~m}, 5 \mathrm{H})$, $3.60(\mathrm{~m}, 1 \mathrm{H}, \mathrm{H}-5), 3.36\left(\mathrm{~s}, 3 \mathrm{H}, \mathrm{OCH}_{3}\right), 2.17(\mathrm{~s}, 3 \mathrm{H}$, $\left.\mathrm{CH}_{3} \mathrm{CO}\right), 2.15\left(\mathrm{~s}, 3 \mathrm{H}, \mathrm{CH}_{3} \mathrm{CO}\right), 1.52$ (s, $6 \mathrm{H}$, isopropylidene), $1.40(\mathrm{~s}, 3 \mathrm{H}$, isopropylidene), $1.29(\mathrm{~s}, 3 \mathrm{H}$, isopropylidene); ${ }^{13} \mathrm{C}$ NMR $\left(100 \mathrm{MHz}, \mathrm{CDCl}_{3}\right): 170.0$, $169.6\left(2 \mathrm{C}, 2 \mathrm{COCH}_{3}\right), 101.4\left(1 \mathrm{C}, \mathrm{Me}_{2} \mathrm{CO}_{2}\right), 100.1$, $100.0(2 \mathrm{C}, 2 \mathrm{C}-1), 99.5\left(1 \mathrm{C}, \mathrm{Me}_{2} \mathrm{CO}_{2}\right), 74.1,71.6,71.1$, $70.0,68.9,68.6,65.4,64.2,62.4,62.2(10 \mathrm{C}, \mathrm{C} 2 \sim 6$, $\left.\mathrm{C} 2^{\prime} \sim 6^{\prime}\right), 54.9\left(\mathrm{OCH}_{3}\right), 29.1,29.0,20.8,20.7$ [4 C, 2 $\left.\left(\mathrm{CH}_{3}\right)_{2} \mathrm{CO}_{2}\right], 19.3,19.1\left(2 \mathrm{C}, 2 \mathrm{COCH}_{3}\right)$. Anal. Calcd for $\mathrm{C}_{23} \mathrm{H}_{36} \mathrm{O}_{13}$ : C, 53.07; H, 6.97. Found: C, 53.32; H, 6.70.

\subsection{Methyl 2,3-di- $O$-acetyl-4,6- $O$-isopropylidene- $\alpha$-D- mannopyranosyl-(1 $\rightarrow 3)$-2- $O$-acetyl-4,6- $O$ - isopropylidene- $\alpha$-D-mannopyranoside (5)}

To a solution of 4 (104 mg, $0.2 \mathrm{mmol})$ in pyridine $(5 \mathrm{~mL})$ was added acetic anhydride $(2.0 \mathrm{~mL}, 2 \mathrm{mmol})$. The reaction mixture was stirred at room temperature (rt) for $12 \mathrm{~h}$, at the end of which time TLC (3:1 petroleum ether-EtOAc) indicated that the reaction was complete. The reaction mixture was concentrated, and then the residue was purified by flash column chromatography on a silica gel column (3:1 petroleum ether-EtOAc) to give compound $\mathbf{5}(100 \mathrm{mg}, 89.3 \%)$ as a foamy solid: $[\alpha]_{\mathrm{D}}$ $+38.9^{\circ}\left(\mathrm{c} 1.5, \mathrm{CHCl}_{3}\right) ;{ }^{1} \mathrm{H} \mathrm{NMR}\left(400 \mathrm{MHz}, \mathrm{CDCl}_{3}\right): \delta$ $5.37\left(\mathrm{dd}, 1 \mathrm{H}, J_{1,2} 1.2 \mathrm{~Hz}, J_{2,3} 3.3 \mathrm{~Hz}, \mathrm{H}-2^{\prime}\right), 5.34(\mathrm{dd}, 1$ $\left.\mathrm{H}, J_{1,2} 1.5, J_{2,3} 3.0 \mathrm{~Hz}, \mathrm{H}-2\right), 5.26\left(\mathrm{dd}, 1 \mathrm{H}, J_{2,3} 3.3, J_{3,4}\right.$ $\left.9.8 \mathrm{~Hz}, \mathrm{H}-3^{\prime}\right), 5.12$ (d, $\left.1 \mathrm{H}, J_{1,2} 1.2 \mathrm{~Hz}, \mathrm{H}-1^{\prime}\right), 4.70$ (d, 1 $\left.\mathrm{H}, J_{1,2} 1.5 \mathrm{~Hz}, \mathrm{H}-1\right), 4.20\left(\mathrm{dd}, 1 \mathrm{H}, J_{3,4}=J_{4,5}=9.9 \mathrm{~Hz}\right.$, H-4), 4.11-3.87 (m, 8 H), 3.66 (m, 1 H, H-5), 3.34 (s, 3 $\left.\mathrm{H}, \mathrm{OCH}_{3}\right), 2.21$ (s, $\left.3 \mathrm{H}, \mathrm{CH}_{3} \mathrm{CO}\right), 2.19$ (s, $3 \mathrm{H}, \mathrm{CH}_{3} \mathrm{CO}$ ), $2.14\left(\mathrm{~s}, 3 \mathrm{H}, \mathrm{CH}_{3} \mathrm{CO}\right), 1.55(\mathrm{~s}, 3 \mathrm{H}$, isopropylidene), 1.53 (s, $3 \mathrm{H}$, isopropylidene), $1.38(\mathrm{~s}, 3 \mathrm{H}$, isopropylidene), 1.31 (s, $3 \mathrm{H}$, isopropylidene). Anal. Calcd for $\mathrm{C}_{25} \mathrm{H}_{38} \mathrm{O}_{14}$ : C, 53.37; H, 6.81. Found: $\mathrm{C}, 53.41 ; \mathrm{H}, 6.62$.

\subsection{Methyl 4,6- $O$-isopropylidene- $\alpha$-D-mannopyranosyl- $(1 \rightarrow 3)-4,6-O$-isopropylidene- $\alpha$-D-mannopyranoside (6)}

Disaccharide 4 (2.60 $\mathrm{mg}, 5.0 \mathrm{mmol})$ was dissolved in a satd solution of ammonia in methanol $(25 \mathrm{~mL})$. After 2 $\mathrm{h}$ at $\mathrm{rt}$, the reaction mixture was concentrated, and the residue was purified by flash column chromatography on a silica gel column (EtOAc) to give compound $\mathbf{6}$ (2.10 g, 96.3\%) as a foamy solid: $[\alpha]_{\mathrm{D}}+90.6^{\circ}\left(c 0.5, \mathrm{CHCl}_{3}\right)$; ${ }^{1} \mathrm{H}$ NMR $\left(400 \mathrm{MHz}, \mathrm{CDCl}_{3}\right): \delta 5.21\left(\mathrm{~s}, 1 \mathrm{H}, \mathrm{H}-1^{\prime}\right), 4.69$ (s, $1 \mathrm{H}, \mathrm{H}-1), 3.34\left(\mathrm{~s}, 3 \mathrm{H}, \mathrm{CH}_{3} \mathrm{CO}\right), 1.52(\mathrm{~s}, 3 \mathrm{H}$, isopropylidene), $1.49(\mathrm{~s}, 3 \mathrm{H}$, isopropylidene $), 1.43(\mathrm{~s}, 3$ $\mathrm{H}$, isopropylidene), $1.37\left(\mathrm{~s}, 3 \mathrm{H}\right.$, isopropylidene); ${ }^{13} \mathrm{C}$ NMR $\left(100 \mathrm{MHz}, \mathrm{CDCl}_{3}\right): \delta 101.4,101.3(2 \mathrm{C}, 2$ $\mathrm{Me}_{2} \mathrm{CO}_{2}$ ), 100.2, 99.8 (2 C, $\left.2 \mathrm{C}-1\right), 73.6,71.4,71.3$, $71.2,70.9,68.9,64.7,64.3,62.3,62.2(10 \mathrm{C}, \mathrm{C} 2 \sim 6$, $\left.\mathrm{C} 2^{\prime} \sim 6^{\prime}\right), 54.9\left(\mathrm{OCH}_{3}\right), 29.3,29.2,19.4,19.3[4 \mathrm{C}, 2$ $\left(\mathrm{CH}_{3}\right)_{2} \mathrm{CO}_{2}$ ]. Anal. Calcd for $\mathrm{C}_{19} \mathrm{H}_{32} \mathrm{O}_{11}: \mathrm{C}, 52.28 ; \mathrm{H}$, 7.39. Found: C, 52.51; H, 7.67.

\subsection{Methyl 2- $O$-acetyl-3,4,6-tri- $O$-benzoyl- $\alpha$-D- mannopyranosyl-( $(\rightarrow 2)-3,4,6$-tri- $O$-benzoyl- $\alpha$-D- mannopyranosyl-( $1 \rightarrow 3)-4,6-O$-isopropylidene- $\alpha$-D- mannopyranosyl-( $1 \rightarrow 3)-4,6-O$-isopropylidene- $\alpha$-D- mannopyranoside (8)}

To a cooled solution $\left(-20^{\circ} \mathrm{C}\right)$ of $6(2.18 \mathrm{~g}, 5 \mathrm{mmol})$ and $7(6.34 \mathrm{~g}, 5.5 \mathrm{mmol})$ in anhyd $\mathrm{CH}_{2} \mathrm{Cl}_{2}(50 \mathrm{~mL})$ was added TMSOTf $(18 \mu \mathrm{L}, 0.05 \mathrm{mmol})$. The mixture was stirred at this temperature for $2 \mathrm{~h}$ and then quenched with $\mathrm{Et}_{3} \mathrm{~N}$ (two drops). The solvents were evaporated in vacuo to give a residue, which was purified by silica gel column chromatography (1:1 petroleum ether-EtOAc) to give trisaccharide $\mathbf{8}(5.08 \mathrm{~g}, 71.2 \%)$ as a syrup: $[\alpha]_{\mathrm{D}}+$ $41.0^{\circ}$ (c 1.0, $\left.\mathrm{CHCl}_{3}\right) ;{ }^{1} \mathrm{H}$ NMR $\left(\mathrm{CDCl}_{3}, 400 \mathrm{MHz}\right): \delta$ $8.05-7.33(\mathrm{~m}, 30 \mathrm{H}, 6 \mathrm{Ph} H), 5.98\left(\mathrm{dd}, 1 \mathrm{H}, J_{2,3} 3.1, J_{3,4}\right.$ $9.8 \mathrm{~Hz}, \mathrm{H}-3), 5.96\left(\mathrm{dd}, 1 \mathrm{H}, J_{3,4}=J_{4,5}=9.8 \mathrm{~Hz}, \mathrm{H}-4\right)$, $5.88\left(\mathrm{dd}, 1 \mathrm{H}, J_{2,3} 3.1, J_{3,4} 9.9 \mathrm{~Hz}, \mathrm{H}-3\right), 5.85(\mathrm{dd}, 1 \mathrm{H}$, $\left.J_{3,4}=J_{4,5}=9.9 \mathrm{~Hz}, \mathrm{H}-4\right), 5.66\left(\mathrm{~d}, 1 \mathrm{H}, J_{1,2} 1.5 \mathrm{~Hz}, \mathrm{H}-1\right)$, $5.64\left(\mathrm{dd}, 1 \mathrm{H}, J_{1,2} 1.6, J_{2,3} 3.1 \mathrm{~Hz}, \mathrm{H}-2\right), 5.36\left(\mathrm{~d}, 1 \mathrm{H}, J_{1,2}\right.$ $0.8 \mathrm{~Hz}, \mathrm{H}-1), 5.13$ (d, $\left.1 \mathrm{H}, J_{1,2} 1.4 \mathrm{~Hz}, \mathrm{H}-1\right), 4.72(\mathrm{~d}, 1 \mathrm{H}$, $\left.J_{1,2} 1.0 \mathrm{~Hz}, \mathrm{H}-1\right), 3.37\left(\mathrm{~s}, 3 \mathrm{H}, \mathrm{OCH}_{3}\right), 2.03(\mathrm{~s}, 3 \mathrm{H}$, $\left.\mathrm{COCH}_{3}\right), 1.49,1.46,1.39,1.34(4 \mathrm{~s}, 12 \mathrm{H}$, isopropylidene); $\left.{ }^{13} \mathrm{C} \mathrm{NMR} \mathrm{(100} \mathrm{MHz,} \mathrm{CDCl}_{3}\right): \delta 169.3\left(\mathrm{COCH}_{3}\right)$, 166.5, 166.1, 165.6, 165.4,165.2, 164.9 (6 C, $6 \mathrm{COPh})$, 101.2, 100.6 (2 C, $\left.2 \mathrm{Me}_{2} \mathrm{C}\right), 99.8,99.5,99.3,99.1$ (4 C, 4 C-1), $54.8\left(\mathrm{OCH}_{3}\right), 29.2,29.1\left(2 \mathrm{C}, \mathrm{CH}_{3} \mathrm{CCH}_{3}\right), 20.5$ 
$\left(\mathrm{COCH}_{3}\right), 19.3,19.2\left(2 \mathrm{C}, \mathrm{CH}_{3} \mathrm{CCH}_{3}\right)$. Anal. Calcd for $\mathrm{C}_{75} \mathrm{H}_{78} \mathrm{O}_{28}$ : C, 63.10; H, 5.51. Found: C, 63.35; H, 5.69.

3.7. Methyl 2- $O$-acetyl-3,4,6-tri- $O$-benzoyl- $\alpha$-Dmannopyranosyl-( $1 \rightarrow 2)-3,4,6$-tri- $O$-benzoyl- $\alpha$-Dmannopyranosyl-( $1 \rightarrow 3)$-2- $O$-acetyl-4,6-O isopropylidene- $\alpha$-D-mannopyranosyl-( $1 \rightarrow 3)$-2- $O$-acetyl4,6- $O$-isopropylidene- $\alpha$-D-mannopyranoside (9)

To a solution of $8(2.85 \mathrm{~g}, 2.0 \mathrm{mmol})$ in pyridine $(30 \mathrm{~mL})$ was added acetic anhydride $(20 \mathrm{~mL}, 20 \mathrm{mmol})$. The reaction mixture was stirred at $\mathrm{rt}$ for $12 \mathrm{~h}$, at the end of which time TLC (3:1 petroleum ether-EtOAc) indicated that the reaction was complete. The reaction mixture was concentrated, and then the residue was purified by flash column chromatography on a silica gel column (3:1 petroleum ether-EtOAc) to give compound 9 (2.81 g, 93.7\%) as a foamy solid: $[\alpha]_{\mathrm{D}}+42.6^{\circ}\left(c 1.0, \mathrm{CHCl}_{3}\right)$; ${ }^{1} \mathrm{H}$ NMR $\left(\mathrm{CDCl}_{3}, 400 \mathrm{MHz}\right): \delta 8.13-7.29(\mathrm{~m}, 30 \mathrm{H}, 6$ $\mathrm{Ph} H), 6.14\left(\mathrm{dd}, 1 \mathrm{H}, J_{3,4}=J_{4,5}=9.9 \mathrm{~Hz}, \mathrm{H}-4\right), 5.99(\mathrm{dd}$, $\left.1 \mathrm{H}, J_{3,4}=J_{4,5}=9.9 \mathrm{~Hz}, \mathrm{H}-4\right), 5.83\left(\mathrm{dd}, 1 \mathrm{H}, J_{2,3} 2.7\right.$, $\left.J_{3,4} 9.9 \mathrm{~Hz}, \mathrm{H}-3\right), 5.72\left(\mathrm{dd}, 1 \mathrm{H}, J_{2,3} 2.4, J_{3,4} 9.9 \mathrm{~Hz}, \mathrm{H}-\right.$ 3), $5.66\left(\mathrm{dd}, 1 \mathrm{H}, J_{1,2} 0.7, J_{2,3} 2.7 \mathrm{~Hz}, \mathrm{H}-2\right), 5.49(\mathrm{~d}, 1 \mathrm{H}$, $\left.J_{1,2} 0.7, \mathrm{H}-1\right), 5.41\left(\mathrm{dd}, 1 \mathrm{H}, J_{1,2} 0.8, J_{2,3} 3.0 \mathrm{~Hz}, \mathrm{H}-2\right)$, $5.22\left(\mathrm{~d}, 1 \mathrm{H}, J_{1,2} 0.7 \mathrm{~Hz}, \mathrm{H}-1\right), 5.11\left(\mathrm{dd}, 1 \mathrm{H}, J_{1,2} 1.0, J_{2,3}\right.$ $3.0 \mathrm{~Hz}, \mathrm{H}-2), 5.10\left(\mathrm{~d}, 1 \mathrm{H}, J_{1,2} 1.0 \mathrm{~Hz}, \mathrm{H}-1\right), 4.64(\mathrm{~d}, 1 \mathrm{H}$, $\left.J_{1,2} 0.8 \mathrm{~Hz}, \mathrm{H}-1\right), 3.36$ (s, $3 \mathrm{H}, \mathrm{OCH}_{3}$ ), 2.32, 2.18, 2.01 (3 $\left.\mathrm{s}, 9 \mathrm{H}, 3 \mathrm{COCH}_{3}\right), 1.53,1.49,1.38,1.32(4 \mathrm{~s}, 12 \mathrm{H}$, isopropylidene); ${ }^{13} \mathrm{C}$ NMR $\left(100 \mathrm{MHz}, \mathrm{CDCl}_{3}\right): \delta 170.2$, $170.2,169.9$ (3 C, $\left.3 \mathrm{COCH}_{3}\right), 166.4,165.8,165.5$, 165.3,165.0, 164.9 (6 C, $6 \mathrm{COPh}), 99.9,99.7$ (2 C, 2 $\left.\mathrm{Me}_{2} \mathrm{C}\right), 99.8,99.4,99.3,99.0$ (4 C, $\left.4 \mathrm{C}-1\right), 55.0\left(\mathrm{OCH}_{3}\right)$, 29.1, $29.0\left(2 \mathrm{C}, \mathrm{CH}_{3} \mathrm{CCH}_{3}\right), 20.9,20.9,20.5$ (3 C, 3 $\left.\mathrm{COCH}_{3}\right), 19.4,19.2\left(2 \mathrm{C}, \mathrm{CH}_{3} \mathrm{CCH}_{3}\right)$. Anal. Calcd for $\mathrm{C}_{79} \mathrm{H}_{82} \mathrm{O}_{30}$ : C, 62.77; H, 5.47. Found: $\mathrm{C}, 62.48 ; \mathrm{H}, 5.33$.

\subsection{Methyl 2- $O$-acetyl-3,4,6-tri- $O$-benzoyl- $\alpha$-D- mannopyranosyl-( $1 \rightarrow 2)-3,4,6$-tri- $O$-benzoyl- $\alpha$-D- mannopyranosyl-( $(\rightarrow 3)$-2- $O$-acetyl- $\alpha$-D- mannopyranosyl-(1 $\rightarrow 3)$-2- $O$-acetyl- $\alpha$-D- mannopyranoside (10)}

Compound 9 (1.51 g, $1.0 \mathrm{mmol})$ was dissolved in $90 \%$ TFA $(20 \mathrm{~mL})$ and stirred for $2 \mathrm{~h}$, at the end of which time the reaction mixture was poured directly into toluene $(100 \mathrm{~mL})$, and then the mixture was concentrated. The residue was purified by flash chromatography (EtOAc) to give $\mathbf{1 0}(1.19 \mathrm{~g}, 83.2 \%)$ as a syrup: $[\alpha]_{\mathrm{D}}$ $+30.1^{\circ}\left(c 1.0, \mathrm{CHCl}_{3}\right) ;{ }^{1} \mathrm{H} \mathrm{NMR}\left(\mathrm{CDCl}_{3}, 400 \mathrm{MHz}\right): \delta$ $7.95-7.25(\mathrm{~m}, 30 \mathrm{H}, 6 \mathrm{Ph} H), 5.95\left(\mathrm{dd}, 1 \mathrm{H}, J_{3,4}=J_{4,5}=\right.$ $9.7 \mathrm{~Hz}, \mathrm{H}-4), 5.88\left(\mathrm{dd}, 1 \mathrm{H}, J_{3,4}=J_{4,5}=9.8 \mathrm{~Hz}, \mathrm{H}-4\right)$, $5.84\left(\mathrm{dd}, 1 \mathrm{H}, J_{2,3} 3.2, J_{3,4} 9.8 \mathrm{~Hz}, \mathrm{H}-3\right), 5.68(\mathrm{dd}, 1 \mathrm{H}$, $\left.J_{2,3} 3.0, J_{3,4} 9.7 \mathrm{~Hz}, \mathrm{H}-3\right), 5.63\left(\mathrm{dd}, 1 \mathrm{H}, J_{1,2} 1.4, J_{2,3} 3.1\right.$ $\mathrm{Hz}, \mathrm{H}-2), 5.56$ (d, $\left.1 \mathrm{H}, J_{1,2} 1.4 \mathrm{~Hz}, \mathrm{H}-1\right), 5.50(\mathrm{dd}, 1 \mathrm{H}$,
$\left.J_{1,2} 1.5, J_{2,3} 3.1 \mathrm{~Hz}, \mathrm{H}-2\right), 5.20\left(\mathrm{dd}, 1 \mathrm{H}, J_{1,2} 1.0, J_{2,3} 3.1\right.$ $\mathrm{Hz}, \mathrm{H}-2), 5.12\left(\mathrm{~d}, 1 \mathrm{H}, J_{1,2} 1.5 \mathrm{~Hz}, \mathrm{H}-1\right), 5.09(\mathrm{~d}, 1 \mathrm{H}$, $\left.J_{1,2} 0.8 \mathrm{~Hz}, \mathrm{H}-1\right), 4.67$ (d, $\left.1 \mathrm{H}, J_{1,2} 0.9 \mathrm{~Hz}, \mathrm{H}-1\right), 3.36$ (s, $\left.3 \mathrm{H}, \mathrm{OCH}_{3}\right), 2.15,2.11,2.00\left(3 \mathrm{~s}, 9 \mathrm{H}, 3 \mathrm{COCH}_{3}\right) ;{ }^{13} \mathrm{C}$ NMR $\left(100 \mathrm{MHz}, \mathrm{CDCl}_{3}\right): \delta 170.7,170.2,169.2(3 \mathrm{C}, 3$ $\left.\mathrm{COCH}_{3}\right), 167.2,166.8,165.5,165.4,165.3,165.0(6 \mathrm{C}, 6$ COPh), 100.5, 99.9, 98.7, 98.4 (4 C, 4 C-1), 55.0 $\left(\mathrm{OCH}_{3}\right), 21.0,20.8,20.5\left(3 \mathrm{C}, 3 \mathrm{COCH}_{3}\right)$. Anal. Calcd for $\mathrm{C}_{73} \mathrm{H}_{74} \mathrm{O}_{30}$ : C, 61.25; H, 5.21. Found: $\mathrm{C}, 61.45 ; \mathrm{H}$, 5.40 .

\subsection{Methyl 2- $O$-acetyl-3,4,6-tri- $O$-benzoyl- $\alpha$-D- mannopyranosyl-( $1 \rightarrow 2)-3,4,6$-tri- $O$-benzoyl- $\alpha$-D- mannopyranosyl-( $1 \rightarrow 3)$-[2- $O$-acetyl-3,4,6-tri- $O$-benzoyl- $\alpha$-D-mannopyranosyl-( $(1 \rightarrow 2)-3,4,6$-tri- $O$-benzoyl- $\alpha$-D- mannopyranosyl- $(1 \rightarrow 6)]-2-O$-acetyl- $\alpha$-D- mannopyranosyl-( $1 \rightarrow 3)$-[2- $O$-acetyl-3,4,6-tri- $O$-benzoyl- $\alpha$-D-mannopyranosyl-( $(1 \rightarrow 2)-3,4,6$-tri- $O$-benzoyl- $\alpha$-D- mannopyranosyl- $(1 \rightarrow 6) \mid-2-O$-acetyl- $\alpha$-D- mannopyranoside (11)}

Compound 10 (715 mg, $0.5 \mathrm{mmol})$ and 7 (1.15 g, 1.0 $\mathrm{mmol}$ ) were dried together under high vacuum for $2 \mathrm{~h}$, then dissolved in anhyd $\mathrm{CH}_{2} \mathrm{Cl}_{2}(50 \mathrm{~mL})$. TMSOTf $(18.0$ $\mu \mathrm{L}, 0.10 \mathrm{mmol}$ ) was added dropwise at $-20^{\circ} \mathrm{C}$ with $\mathrm{N}_{2}$ protection. The reaction mixture was stirred for $2 \mathrm{~h}$, during which time the temperature was gradually warmed to ambient temperature. Then the mixture was neutralized with $\mathrm{Et}_{3} \mathrm{~N}$ and concentrated to dryness under reduced pressure. Purification of the residue by column chromatography on a silica gel column (1:1 petroleum ether-EtOAc) furnished the octasaccharide $11(1.03 \mathrm{~g}, 60.9 \%)$ as a syrup: $[\alpha]_{\mathrm{D}}+28.5^{\circ}$ (c 1.0, $\left.\mathrm{CHCl}_{3}\right) ;{ }^{1} \mathrm{H} \mathrm{NMR}\left(\mathrm{CDCl}_{3}, 400 \mathrm{MHz}\right): \delta 8.09-7.25(\mathrm{~m}$, $90 \mathrm{H}, 18 \mathrm{PhH}), 6.04-5.84(\mathrm{~m}, 10 \mathrm{H}), 5.73-5.67(\mathrm{~m}, 5$ $\mathrm{H}), 5.61\left(\mathrm{dd}, 1 \mathrm{H}, J_{1,2} 1.0, J_{2,3} 3.1 \mathrm{~Hz}, \mathrm{H}-2\right), 5.34(\mathrm{~d}, 1 \mathrm{H}$, $\left.J_{1,2} 0.7 \mathrm{~Hz}, \mathrm{H}-1\right), 5.30\left(\mathrm{dd}, 1 \mathrm{H}, J_{1,2} 1.1, J_{2,3} 3.2 \mathrm{~Hz}, \mathrm{H}-\right.$ 2), $5.27\left(\mathrm{~d}, 1 \mathrm{H}, J_{1,2} 1.1 \mathrm{~Hz}, \mathrm{H}-1\right), 5.24\left(\mathrm{~d}, 1 \mathrm{H}, J_{1,2} 1.0\right.$ $\mathrm{Hz}, \mathrm{H}-1), 5.14$ (d, $\left.1 \mathrm{H}, J_{1,2} 1.2 \mathrm{~Hz}, \mathrm{H}-1\right), 5.08(\mathrm{~d}, 1 \mathrm{H}$, $\left.J_{1,2} 1.2 \mathrm{~Hz}, \mathrm{H}-1\right), 5.07$ (d, $\left.1 \mathrm{H}, J_{1,2} 0.8 \mathrm{~Hz}, \mathrm{H}-1\right), 4.86-$ $4.75(\mathrm{~m}, 2 \mathrm{H}), 4.70$ (d, $\left.1 \mathrm{H}, J_{1,2} 0.8 \mathrm{~Hz}, \mathrm{H}-1\right), 4.61$ (d, 1 $\left.\mathrm{H}, J_{1,2} 0.9 \mathrm{~Hz}, \mathrm{H}-1\right), 3.35\left(\mathrm{~s}, 3 \mathrm{H}, \mathrm{OCH}_{3}\right), 2.16,2.12$, 2.01, 1.99, $1.96\left(5 \mathrm{~s}, 15 \mathrm{H}, 5 \mathrm{COCH}_{3}\right) ;{ }^{13} \mathrm{C}$ NMR (100 $\left.\mathrm{MHz}, \mathrm{CDCl}_{3}\right): \delta 170.5,170.2,169.2,169.2,169.1$ (5 C, 5 $\left.\mathrm{COCH}_{3}\right), 167.2,166.5,165.4,165.3,165.2,165.9,165.6$, $165.6,165.5,165.5,165.4,165.3,165.3,165.2,165.2$, 165.1, 165.0, 164.9 (18 C, $18 \mathrm{COPh}), 100.6,100.0,99.8$, 99.7, 99.4, 98.9, 98.6, $98.3(8 \mathrm{C}, 8 \mathrm{C}-1), 54.9\left(\mathrm{OCH}_{3}\right)$, 21.0, 20.8, 20.5 (5 C, $5 \mathrm{COCH}_{3}$ ). Anal. Calcd for $\mathrm{C}_{185} \mathrm{H}_{166} \mathrm{O}_{64}: \mathrm{C}, 65.09 ; \mathrm{H}, 4.90$. Found: $\mathrm{C}, 65.25 ; \mathrm{H}$, 4.79 . 
3.10. Methyl 2- $O$-acetyl-3,4,6-tri- $O$-benzoyl- $\alpha$-Dmannopyranosyl-( $1 \rightarrow 2)-3,4,6$-tri- $O$-benzoyl- $\alpha$-Dmannopyranosyl-( $1 \rightarrow 3)$-[2- $O$-acetyl-3,4,6-tri- $O$-benzoyl$\alpha$-D-mannopyranosyl-( $1 \rightarrow 2)-3,4,6$-tri- $O$-benzoyl- $\alpha$-Dmannopyranosyl-( $(1 \rightarrow 6)]-2,4-$ di- $O$-acetyl- $\alpha$-Dmannopyranosyl-( $(\rightarrow 3)$-[2- $O$-acetyl-3,4,6-tri- $O$-benzoyl$\alpha$-D-mannopyranosyl-( $1 \rightarrow 2)-3,4,6$-tri- $O$-benzoyl- $\alpha$-Dmannopyranosyl-( $1 \rightarrow 6)]$-2,4-di- $O$-acetyl- $\alpha$-Dmannopyranoside (12)

To a solution of $11(1.02 \mathrm{~g}, 0.3 \mathrm{mmol})$ in pyridine (20 $\mathrm{mL})$ was added acetic anhydride $(10 \mathrm{~mL}, 10 \mathrm{mmol})$. The reaction mixture was stirred at $\mathrm{rt}$ for $12 \mathrm{~h}$, at the end of which time TLC $(1: 1$ petroleum ether-EtOAc) indicated that the reaction was complete. The reaction mixture was concentrated, and then the residue was purified by flash column chromatography on a silica gel column (1:1 petroleum ether-EtOAc) to give compound 12 (964 $\mathrm{mg}, 91.8 \%)$ as a foamy solid: $[\alpha]_{\mathrm{D}}+33.0^{\circ}$ (c 0.3 , $\left.\mathrm{CHCl}_{3}\right) ;{ }^{1} \mathrm{H}$ NMR $\left(\mathrm{CDCl}_{3}, 400 \mathrm{MHz}\right): \delta 6.14(\mathrm{dd}, 1$ $\left.\mathrm{H}, J_{3,4}=J_{4,5}=9.8 \mathrm{~Hz}, \mathrm{H}-4\right), 6.05\left(\mathrm{dd}, 1 \mathrm{H}, J_{3,4}=J_{4,5}=\right.$ $9.9 \mathrm{~Hz}, \mathrm{H}-4), 6.01\left(\mathrm{dd}, 1 \mathrm{H}, J_{3,4}=J_{4,5}=9.8 \mathrm{~Hz}, \mathrm{H}-4\right)$, $5.99\left(\mathrm{dd}, \mathrm{H}, J_{3,4}=J_{4,5}=9.8 \mathrm{~Hz}, \mathrm{H}-4\right), 5.96(\mathrm{dd}, 1 \mathrm{H}$, $\left.J_{3,4}=J_{4,5}=10.0 \mathrm{~Hz}, \mathrm{H}-4\right), 5.91\left(\mathrm{dd}, \mathrm{H}, J_{3,4}=J_{4,5}=9.9\right.$ $\mathrm{Hz}, \mathrm{H}-4), 5.90-5.80(\mathrm{~m}, 5 \mathrm{H}), 5.75\left(\mathrm{dd}, 1 \mathrm{H}, J_{2,3} 3.0, J_{3,4}\right.$ $9.7 \mathrm{~Hz}, \mathrm{H}-3), 5.68-5.64(\mathrm{~m}, 3 \mathrm{H}), 5.38\left(\mathrm{~d}, 1 \mathrm{H}, J_{1,2} 0.7\right.$ $\mathrm{Hz}, \mathrm{H}-1), 5.38\left(\mathrm{dd}, 1 \mathrm{H}, J_{3,4}=J_{4,5}=10.0 \mathrm{~Hz}, \mathrm{H}-4\right), 5.33$ $\left(\mathrm{dd}, 1 \mathrm{H}, J_{1,2} 1.0, J_{2,3} 3.1 \mathrm{~Hz}, \mathrm{H}-2\right), 5.30\left(\mathrm{dd}, 1 \mathrm{H}, J_{3,4}=\right.$ $\left.J_{4,5}=10.0 \mathrm{~Hz}, \mathrm{H}-4\right), 5.24\left(\mathrm{~d}, 2 \mathrm{H}, J_{1,2} 1.4,2 \mathrm{H}-1\right), 5.19$ (d, $\left.1 \mathrm{H}, J_{1,2} 0.8 \mathrm{~Hz}, \mathrm{H}-1\right), 5.14-5.08(\mathrm{~m}, 4 \mathrm{H}, 3 \mathrm{H}-1, \mathrm{H}-$ 2), $4.73\left(\mathrm{~d}, 1 \mathrm{H}, J_{1,2} 1.3 \mathrm{~Hz}, \mathrm{H}-1\right), 3.33\left(\mathrm{~s}, 3 \mathrm{H}, \mathrm{OCH}_{3}\right.$ ), 2.24, 2.16, 2.08, 2.06, 1.99, 1.98, $1.96(7 \mathrm{~s}, 21 \mathrm{H}, 7$ $\mathrm{COCH}_{3}$ ). Anal. Calcd for $\mathrm{C}_{189} \mathrm{H}_{170} \mathrm{O}_{66}: \mathrm{C}, 64.90 ; \mathrm{H}$, 4.90. Found: C, 64.75; H, 4.81.

\subsection{Methyl $\alpha$-D-mannopyranosyl-( $1 \rightarrow 2)-\alpha$-D-} mannopyranosyl-( $1 \rightarrow 3)$-[ $\alpha$-D-mannopyranosyl-(1 $\rightarrow 2)-\alpha$ D-mannopyranosyl-(1 $\rightarrow$ 6)]- $\alpha$-D-mannopyranosyl-(1 $\rightarrow 3)$ [ $\alpha$-D-mannopyranosyl-(1 $\rightarrow 2)$ - $\alpha$-D-mannopyranosyl- $(1 \rightarrow$ 6)]- $\alpha$-D-mannopyranoside (13)

Octasaccharide 12 (700 mg, $0.20 \mathrm{mmol}$ ) was dissolved in a saturated solution of ammonia in $\mathrm{MeOH}(30 \mathrm{~mL})$. After $96 \mathrm{~h}$ at rt, the reaction mixture was concentrated, and the residue was purified by chromatography on Sephadex LH-20 $\left(\mathrm{H}_{2} \mathrm{O}\right)$ to afford $\mathbf{1 3}(193 \mathrm{mg}, 72.6 \%)$ as a foamy solid: ${ }^{1} \mathrm{H}$ NMR $\left(\mathrm{D}_{2} \mathrm{O}, 400 \mathrm{MHz}\right): \delta 5.24$ (s, 1 $\mathrm{H}, \mathrm{H}-1), 5.02$ (s, $2 \mathrm{H}, 2 \mathrm{H}-1), 4.91\left(\mathrm{~d}, 2 \mathrm{H}, J_{1,2} 1.2 \mathrm{~Hz}, 2\right.$ H-1), 4.90 (s, 1 H, H-1), 4.87 (s, 1 H, H-1), 4.61 (s, $1 \mathrm{H}$, $\mathrm{H}-1), 3.29$ (s, $\left.3 \mathrm{H}, \mathrm{OCH}_{3}\right) ;{ }^{13} \mathrm{C}$ NMR $\left(100 \mathrm{MHz}, \mathrm{D}_{2} \mathrm{O}\right)$ : $\delta$ 105.1, 105.1, 104.9, 104.9, 104.9, 103.6, 103.4, 100.6, $100.5(8 \mathrm{C}, 8 \mathrm{C}-1), 57.5\left(\mathrm{OCH}_{3}\right)$. MALDI-TOF MS Calcd for $\mathrm{C}_{49} \mathrm{H}_{84} \mathrm{O}_{41}: 1329.2$ [M]. Found: $1352.1[\mathrm{M}+$ $\left.\mathrm{Na}^{+}\right]$.
3.12. Methyl 2- $O$-acetyl-3,4,6-tri- $O$-benzoyl- $\alpha$-Dmannopyranosyl-( $1 \rightarrow 3)-4,6-O$-isopropylidene- $\alpha$-Dmannopyranosyl-( $1 \rightarrow 3)-4,6-O$-isopropylidene- $\alpha$-Dmannopyranoside (15)

To a cooled solution $\left(-20^{\circ} \mathrm{C}\right)$ of $\mathbf{6}(2.18 \mathrm{~g}, 5 \mathrm{mmol})$ and $14(3.73 \mathrm{~g}, 5.5 \mathrm{mmol})$ in anhyd $\mathrm{CH}_{2} \mathrm{Cl}_{2}(50 \mathrm{~mL})$ was added TMSOTf $(18 \mu \mathrm{L}, 0.05 \mathrm{mmol})$. The mixture was stirred at this temperature for $2 \mathrm{~h}$, and then quenched with $\mathrm{Et}_{3} \mathrm{~N}$ (two drops). The solvents were evaporated in vacuo to give a residue, which was purified by silica gel column chromatography (1:1 petroleum ether-EtOAc) to give trisaccharide $15(3.20 \mathrm{~g}, 67.2 \%)$ as a syrup: $[\alpha]_{\mathrm{D}}$ $+91.7^{\circ}\left(c\right.$ 1.0, $\left.\mathrm{CHCl}_{3}\right) ;{ }^{1} \mathrm{H}$ NMR $\left(\mathrm{CDCl}_{3}, 400 \mathrm{MHz}\right): \delta$ $7.95-7.34(\mathrm{~m}, 15 \mathrm{H}, 3 \mathrm{Ph} H), 5.94\left(\mathrm{dd}, 1 \mathrm{H}, J_{3,4}=J_{4,5}=\right.$ $10.0 \mathrm{~Hz}, \mathrm{H}-4), 5.75$ (dd, $\left.1 \mathrm{H}, J_{2,3} 3.3, J_{3,4} 10.0 \mathrm{~Hz}, \mathrm{H}-3\right)$, $5.53\left(\mathrm{dd}, 1 \mathrm{H}, J_{1,2} 1.8, J_{2,3} 3.3 \mathrm{~Hz}, \mathrm{H}-2\right), 5.43\left(\mathrm{~d}, 1 \mathrm{H}, J_{1,2}\right.$ $1.8 \mathrm{~Hz}, \mathrm{H}-1), 5.31\left(\mathrm{~d}, 1 \mathrm{H}, J_{1,2} 1.0 \mathrm{~Hz}, \mathrm{H}-1\right), 4.71(\mathrm{~d}, 1 \mathrm{H}$, $\left.J_{2,1} 1.0 \mathrm{~Hz}, \mathrm{H}-1\right), 4.66-4.58(\mathrm{~m}, 3 \mathrm{H}), 4.26-4.18(\mathrm{~m}, 4$ H), 4.04-3.99 (m, $2 \mathrm{H}), 3.90-3.82(\mathrm{~m}, 4 \mathrm{H}), 3.68-3.63$ $(\mathrm{m}, 2 \mathrm{H}), 3.36\left(\mathrm{~s}, 3 \mathrm{H}, \mathrm{OCH}_{3}\right), 2.17\left(\mathrm{~s}, 3 \mathrm{H}, \mathrm{COCH}_{3}\right)$, 1.56, $1.45(2 \mathrm{~s}, 6 \mathrm{H}$, isopropylidene $), 1.37(\mathrm{~s}, 6 \mathrm{H}$, isopropylidene); ${ }^{13} \mathrm{C}$ NMR (100 $\left.\mathrm{MHz}, \mathrm{CDCl}_{3}\right): \delta 169.5$ $\left(\mathrm{COCH}_{3}\right), 166.3,165.6,163.6(5 \mathrm{C}, 5 \mathrm{COPh}), 101.2$, 100.8, (2 C, $\left.2 \mathrm{Me}_{2} C\right)$ 99.9, 99.4, 98.3 (3 C, $\left.3 \mathrm{C}-1\right), 54.8$ $\left(\mathrm{OCH}_{3}\right), 29.1,28.9\left(2 \mathrm{C}, \mathrm{CH}_{3} \mathrm{CCH}_{3}\right), 20.6\left(\mathrm{COCH}_{3}\right)$, 19.2, $19.0\left(2 \mathrm{C}, \mathrm{CH}_{3} \mathrm{CCH}_{3}\right)$. Anal. Calcd for $\mathrm{C}_{48} \mathrm{H}_{56} \mathrm{O}_{20}$. C, 60.50; H, 5.92. Found: C, 60.45; H, 5.66.

\subsection{Methyl 2- $O$-acetyl-3,4,6-tri- $O$-benzoyl- $\alpha$-D- mannopyranosyl-( $1 \rightarrow 3)-2-O$-acetyl-4,6- $O$ - isopropylidene- $\alpha$-D-mannopyranosyl-( $1 \rightarrow 3)-2-O$-acetyl- 4,6- $O$-isopropylidene- $\alpha$-D-mannopyranoside (16)}

To a solution of $\mathbf{1 5}(950 \mathrm{mg}, 1.0 \mathrm{mmol})$ in pyridine $(50$ $\mathrm{mL}$ ) was added acetic anhydride $(20 \mathrm{~mL}, 20 \mathrm{mmol})$. The reaction mixture was stirred at $\mathrm{rt}$ for $12 \mathrm{~h}$, at the end of which time TLC (3:1 petroleum ether-EtOAc) indicated that the reaction was complete. The reaction mixture was concentrated, and then the residue was purified by flash column chromatography on a silica gel column (3:1 petroleum ether-EtOAc) to give compound 16 (830 $\mathrm{mg}, 80.6 \%)$ as a foamy solid: $[\alpha]_{\mathrm{D}}+82.6^{\circ}$ (c 1.0, $\left.\mathrm{CHCl}_{3}\right) ;{ }^{1} \mathrm{H} \mathrm{NMR}\left(\mathrm{CDCl}_{3}, 400 \mathrm{MHz}\right): \delta$ 8.10-7.23 $(\mathrm{m}, 65 \mathrm{H}, 13 \mathrm{Ph} H), 6.04\left(\mathrm{dd}, 1 \mathrm{H}, J_{3,4}=J_{4,5}=10.0 \mathrm{~Hz}\right.$, H-4), 5.64 (dd, $\left.1 \mathrm{H}, J_{2,3} 3.2, J_{3,4} 10.0 \mathrm{~Hz}, \mathrm{H}-3\right), 5.45$ (dd, $\left.1 \mathrm{H}, J_{1,2} 1.8, J_{2,3} 2.9 \mathrm{~Hz}, \mathrm{H}-2\right), 5.40\left(\mathrm{dd}, 1 \mathrm{H}, J_{1,2} 1.3, J_{2,3}\right.$ $3.4 \mathrm{~Hz}, \mathrm{H}-2), 5.32\left(\mathrm{~d}, 1 \mathrm{H}, J_{1,2} 1.8 \mathrm{~Hz}, \mathrm{H}-1\right), 5.22(\mathrm{dd}, 1$ $\left.\mathrm{H}, J_{1,2} 1.3, J_{2,3} 2.9 \mathrm{~Hz}, \mathrm{H}-2\right), 5.10\left(\mathrm{~d}, 1 \mathrm{H}, J_{1,2} 1.3 \mathrm{~Hz}, \mathrm{H}-\right.$ 1), $4.67\left(\mathrm{dd}, 1 \mathrm{H}, J_{2,3} 2.9, J_{3,4} 12.1 \mathrm{~Hz}, \mathrm{H}-3\right), 4.63(\mathrm{~d}, 1$ $\left.\mathrm{H}, J_{1,2} 1.3 \mathrm{~Hz}, \mathrm{H}-1\right), 4.46-4.38(\mathrm{~m}, 2 \mathrm{H}), 4.09-4.05(\mathrm{~m}, 4$ $\mathrm{H}), 3.87-3.81(\mathrm{~m}, 4 \mathrm{H}), 3.70-3.60(\mathrm{~m}, 2 \mathrm{H}), 3.36(\mathrm{~s}, 3 \mathrm{H}$, $\left.\mathrm{OCH}_{3}\right), 2.31,2.18,2.07\left(3 \mathrm{~s}, 9 \mathrm{H}, 3 \mathrm{COCH}_{3}\right), 1.56,1.52$ ( $2 \mathrm{~s}, 6 \mathrm{H}$, isopropylidene), 1.37 (s, $6 \mathrm{H}$, isopropylidene). Anal. Calcd for $\mathrm{C}_{52} \mathrm{H}_{60} \mathrm{O}_{22}$ : C, 60.22; H, 5.83. Found: $\mathrm{C}$, 60.47; H, 5.61. 
3.14. Methyl 2- $O$-acetyl-3,4,6-tri- $O$-benzoyl- $\alpha-\mathrm{D}-$ mannopyranosyl- $(1 \rightarrow 3)$-2- $O$-acetyl- $\alpha$-Dmannopyranosyl-(1 $\rightarrow 3)$-2- $O$-acetyl- $\alpha$-Dmannopyranoside (17)

Compound 16 (1.04 g, $1 \mathrm{mmol})$ was dissolved in 90\% TFA $(20 \mathrm{~mL})$ and stirred for $2 \mathrm{~h}$, at the end of which time the reaction mixture was poured directly into toluene $(100 \mathrm{~mL})$, and then the mixture was concentrated. The residue was purified by flash chromatography (EtOAc) to give $\mathbf{1 7}(765 \mathrm{mg}, 80.1 \%)$ as a syrup: $[\alpha]_{\mathrm{D}}$ $+35.3^{\circ}\left(c 1.0, \mathrm{CHCl}_{3}\right) ;{ }^{1} \mathrm{H}$ NMR $\left(\mathrm{CDCl}_{3}, 400 \mathrm{MHz}\right): \delta$ 7.97-7.26 (m, $10 \mathrm{H}, 2 \mathrm{Ph} H), 5.94\left(\mathrm{dd}, 1 \mathrm{H}, J_{3,4}=J_{4,5}=\right.$ $9.8 \mathrm{~Hz}, \mathrm{H}-4), 5.61$ (dd, $1 \mathrm{H}, J_{2,3} 3.2, J_{3,4} 9.8 \mathrm{~Hz}, \mathrm{H}-3$ ), $5.51\left(\mathrm{dd}, 1 \mathrm{H}, J_{1,2} 1.0, J_{2,3} 3.2 \mathrm{~Hz}, \mathrm{H}-2\right), 5.13(\mathrm{dd}, 1 \mathrm{H}$, $\left.J_{1,2} 0.7, J_{2,3} 3.0 \mathrm{~Hz}, \mathrm{H}-2\right), 5.35\left(\mathrm{~d}, 1 \mathrm{H}, J_{1,2} 1.0 \mathrm{~Hz}, \mathrm{H}-1\right)$, $5.19\left(\mathrm{dd}, 1 \mathrm{H}, J_{1,2} 0.8, J_{2,3} 3.1 \mathrm{~Hz}, \mathrm{H}-2\right), 5.13\left(\mathrm{~d}, 1 \mathrm{H}, J_{1,2}\right.$ $0.7 \mathrm{~Hz}, \mathrm{H}-1), 4.66$ (d, $\left.1 \mathrm{H}, J_{1,2} 0.8 \mathrm{~Hz}, \mathrm{H}-1\right), 3.36$ (s, $3 \mathrm{H}$, $\left.\mathrm{OCH}_{3}\right), 2.20,2.12,2.09\left(3 \mathrm{~s}, 9 \mathrm{H}, 3 \mathrm{COCH}_{3}\right) ;{ }^{13} \mathrm{C} \mathrm{NMR}$ $\left(100 \mathrm{MHz}, \mathrm{CDCl}_{3}\right): 170.7,170.5,170.0\left(3 \mathrm{C}, 3 \mathrm{COCH}_{3}\right)$, 166.8, 165.5 (2 C, 2 COPh), 99.9, 98.8, 98.5 (3 C, 3 C-1), $55.0\left(\mathrm{OCH}_{3}\right), 20.9,20.8,20.6\left(3 \mathrm{C}, 3 \mathrm{COCH}_{3}\right)$. Anal. Calcd for $\mathrm{C}_{46} \mathrm{H}_{52} \mathrm{O}_{22}: \mathrm{C}, 57.73 ; \mathrm{H}, 5.48$. Found: C, 57.57; H, 5.62.

\subsection{Methyl 2- $O$-acetyl-3,4,6-tri- $O$-benzoyl- $\alpha$-D-} mannopyranosyl-(1 $\rightarrow 3)$-[2- $O$-acetyl-3,4,6-tri- $O$-benzoyl$\alpha$-D-mannopyranosyl-(1 $\rightarrow 2)-3,4,6$-tri- $O$-benzoyl- $\alpha$-Dmannopyranosyl-( $(\rightarrow 6)]-2-O$-acetyl- $\alpha$-Dmannopyranosyl-( $1 \rightarrow 3)$-[2- $O$-acetyl-3,4,6-tri- $O$-benzoyl$\alpha$-D-mannopyranosyl-( $1 \rightarrow 2)$-3,4,6-tri- $O$-benzoyl- $\alpha$-Dmannopyranosyl-( $1 \rightarrow 6)]-2-O$-acetyl- $\alpha-\mathrm{D}-$ mannopyranoside (18)

Compound 17 (478 mg, $0.5 \mathrm{mmol})$ and 7 (1.15 g, 1.0 mmol) were dried together under high vacuum for $2 \mathrm{~h}$, then dissolved in anhyd $\mathrm{CH}_{2} \mathrm{Cl}_{2}(50 \mathrm{~mL})$. TMSOTf $(18.0$ $\mu \mathrm{L}, 0.10 \mathrm{mmol}$ ) was added dropwise at $-20{ }^{\circ} \mathrm{C}$ with $\mathrm{N}_{2}$ protection. The reaction mixture was stirred for $2 \mathrm{~h}$, and was neutralized with $\mathrm{Et}_{3} \mathrm{~N}$. Then the mixture was concentrated to dryness under reduced pressure. Purification of the residue by column chromatography on a silica gel column (1:1 petroleum ether-EtOAc) furnished the heptasaccharide $\mathbf{1 8}(1.10 \mathrm{~g}, 74.8 \%)$ as a syrup: $[\alpha]_{\mathrm{D}}+24.5^{\circ}\left(c 1.0, \mathrm{CHCl}_{3}\right) ;{ }^{1} \mathrm{H}$ NMR $\left(\mathrm{CDCl}_{3}, 400\right.$ MHz): $\delta 8.03-7.26(\mathrm{~m}, 75 \mathrm{H}, 15 \mathrm{Ph} H), 6.00-5.83(\mathrm{~m}, 9$ H), $5.69\left(\mathrm{dd}, 1 \mathrm{H}, J_{2,3} 3.1, J_{3,4} 10.1 \mathrm{~Hz}, \mathrm{H}-3\right), 5.67-5.66$ $(\mathrm{m}, 2 \mathrm{H}), 5.59\left(\mathrm{dd}, 1 \mathrm{H}, J_{1,2} 1.2, J_{2,3} 2.9 \mathrm{~Hz}, \mathrm{H}-2\right), 5.56$ (dd, $\left.1 \mathrm{H}, J_{1,2} 0.8, J_{2,3} 2.7 \mathrm{~Hz}, \mathrm{H}-2\right), 5.41\left(\mathrm{~d}, 1 \mathrm{H}, J_{1,2} 0.8\right.$, $\mathrm{H}-1), 5.33\left(\mathrm{~d}, 1 \mathrm{H}, J_{1,2} 1.0 \mathrm{~Hz}, \mathrm{H}-1\right), 5.28\left(\mathrm{dd}, 1 \mathrm{H}, J_{1,2}\right.$ $\left.1.3, J_{2,3} 3.1 \mathrm{~Hz}, \mathrm{H}-2\right), 5.28\left(\mathrm{~d}, 1 \mathrm{H}, J_{1,2} 1.3 \mathrm{~Hz}, \mathrm{H}-1\right), 5.26$ $\left(\mathrm{d}, 1 \mathrm{H}, J_{1,2} 0.8 \mathrm{~Hz}, \mathrm{H}-1\right), 5.10\left(\mathrm{~d}, 1 \mathrm{H}, J_{1,2} 1.0 \mathrm{~Hz}, \mathrm{H}-1\right)$, $5.07\left(\mathrm{~d}, 1 \mathrm{H}, J_{1,2} 0.8 \mathrm{~Hz}, \mathrm{H}-1\right), 4.71\left(\mathrm{~d}, 1 \mathrm{H}, J_{1,2} 1.0 \mathrm{~Hz}\right.$, $\mathrm{H}-1), 3.33$ (s, $\left.3 \mathrm{H}, \mathrm{OCH}_{3}\right), 2.17,2.11,2.09,2.01,2.00$ (5 s, $\left.15 \mathrm{H}, 5 \mathrm{COCH}_{3}\right) ;{ }^{13} \mathrm{C}$ NMR $\left(100 \mathrm{MHz}, \mathrm{CDCl}_{3}\right): \delta$ $170.4,170.3,169.7,169.2,169.2\left(5 \mathrm{C}, 5 \mathrm{COCH}_{3}\right), 166.8$,
$166.5,166.3,166.2,165.9,165.6,165.6,165.5,165.4$, $165.4,165.3,165.3,165.2,165.0,164.9(15 \mathrm{C}, 15 \mathrm{COPh})$, 100.1, 99.8, 99.7, 98.9, 98.7, 98.6, 98.3 (7 C, 7 C-1), 54.9 $\left(\mathrm{OCH}_{3}\right), 21.8,20.7,20.6,20.5,20.5\left(5 \mathrm{C}, 5 \mathrm{COCH}_{3}\right)$. Anal. Calcd for $\mathrm{C}_{158} \mathrm{H}_{144} \mathrm{O}_{56}: \mathrm{C}, 64.57 ; \mathrm{H}, 4$.94. Found: C, 64.30; H, 4.88.

3.16. Methyl 2- $O$-acetyl-3,4,6-tri- $O$-benzoyl- $\alpha$-Dmannopyranosyl-(1 $\rightarrow 3)$-[2- $O$-acetyl-3,4,6-tri- $O$-benzoyl$\alpha$-D-mannopyranosyl-( $(\rightarrow 2)-3,4,6$-tri- $O$-benzoyl- $\alpha$-Dmannopyranosyl-( $(1 \rightarrow 6)$ )-2,4-di- $O$-acetyl- $\alpha$-Dmannopyranosyl-( $1 \rightarrow 3)$-[2- $O$-acetyl-3,4,6-tri- $O$-benzoyl$\alpha$-D-mannopyranosyl-( $(\rightarrow 2)-3,4,6$-tri- $O$-benzoyl- $\alpha$-Dmannopyranosyl-(1 $\rightarrow 6)$ ]-2,4-di- $O$-acetyl- $\alpha$-Dmannopyranoside (19)

To a solution of $\mathbf{1 8}(880 \mathrm{mg}, 0.3 \mathrm{mmol})$ in pyridine ( 20 $\mathrm{mL})$ was added acetic anhydride $(10 \mathrm{~mL}, 10 \mathrm{mmol})$. The reaction mixture was stirred at $\mathrm{rt}$ for $12 \mathrm{~h}$, at the end of which time TLC (1:1 petroleum ether-EtOAc) indicated that the reaction was complete. The reaction mixture was concentrated, and then the residue was purified by flash column chromatography on a silica gel column (1:1 petroleum ether-EtOAc) to give compound 19 (729 $\mathrm{mg}, 80.5 \%)$ as a foamy solid: $[\alpha]_{\mathrm{D}}+23.2^{\circ}$ (c 0.3 , $\left.\mathrm{CHCl}_{3}\right) ;{ }^{1} \mathrm{H}$ NMR $\left(\mathrm{CDCl}_{3}, 400 \mathrm{MHz}\right): \delta 8.08-7.25$ $(\mathrm{m}, 75 \mathrm{H}, 15 \mathrm{Ph} H), 6.06\left(\mathrm{dd}, 1 \mathrm{H}, J_{3,4}=J_{4,5}=10.0 \mathrm{~Hz}\right.$, $\mathrm{H}-4), 6.05\left(\mathrm{dd}, 1 \mathrm{H}, J_{3,4}=J_{4,5}=9.9 \mathrm{~Hz}, \mathrm{H}-4\right), 6.00-5.85$ $(\mathrm{m}, 6 \mathrm{H}), 5.82\left(\mathrm{dd}, 1 \mathrm{H}, J_{2,3} 3.2, J_{3,4} 10.0 \mathrm{~Hz}, \mathrm{H}-3\right), 5.69$ $\left(\mathrm{dd}, 1 \mathrm{H}, J_{2,3} 3.1, J_{3,4} 9.8 \mathrm{~Hz}, \mathrm{H}-3\right), 5.66\left(\mathrm{dd}, 1 \mathrm{H}, J_{1,2}\right.$ $\left.1.1, J_{2,3} 3.2 \mathrm{~Hz}, \mathrm{H}-2\right), 5.64\left(\mathrm{dd}, 1 \mathrm{H}, J_{1,2} 1.0, J_{2,3} 3.0 \mathrm{~Hz}\right.$, $\mathrm{H}-2), 5.43\left(\mathrm{dd}, 1 \mathrm{H}, J_{3,4}=J_{4,5}=10.0 \mathrm{~Hz}, \mathrm{H}-4\right), 5.34(\mathrm{dd}$, $\left.1 \mathrm{H}, J_{1,2} 1.3, J_{2,3} 3.1 \mathrm{~Hz}, \mathrm{H}-2\right), 5.31\left(\mathrm{dd}, 1 \mathrm{H}, J_{1,2} 1.5, J_{2,3}\right.$ $3.2 \mathrm{~Hz}, \mathrm{H}-2), 5.30\left(\mathrm{dd}, 1 \mathrm{H}, J_{3,4}=J_{4,5}=10.2 \mathrm{~Hz}, \mathrm{H}-4\right)$, $5.23\left(\mathrm{~d}, 2 \mathrm{H}, J_{1,2} 1.3 \mathrm{~Hz}, 2 \mathrm{H}-1\right), 5.20\left(\mathrm{~d}, 2 \mathrm{H}, J_{1,2} 1.4 \mathrm{~Hz}\right.$, $2 \mathrm{H}-1), 5.09$ (dd, $\left.1 \mathrm{H}, J_{1,2} 1.1, J_{2,3} 3.0 \mathrm{~Hz}, \mathrm{H}-2\right), 5.09$ (d, $\left.\mathrm{H}, J_{1,2} 1.3 \mathrm{~Hz}, \mathrm{H}-1\right), 5.06\left(\mathrm{~d}, \mathrm{H}, J_{1,2} 1.1 \mathrm{~Hz}, \mathrm{H}-1\right), 4.74$ $\left(\mathrm{d}, 1 \mathrm{H}, J_{1,2} 1.0 \mathrm{~Hz}, \mathrm{H}-1\right), 3.30\left(\mathrm{~s}, 3 \mathrm{H}, \mathrm{OCH}_{3}\right), 2.23$, 2.16, 2.14, 2.14, 2.09, 2.00, $1.98\left(7 \mathrm{~s}, 21 \mathrm{H}, 7 \mathrm{COCH}_{3}\right)$. Anal. Calcd for $\mathrm{C}_{162} \mathrm{H}_{148} \mathrm{O}_{58}: \mathrm{C}, 64.36 ; \mathrm{H}, 4.94$. Found: C, 64.50; H, 5.13.

\subsection{Methyl $\alpha$-D-mannopyranosyl-( $1 \rightarrow 3)$-[ $\alpha$-D- mannopyranosyl-(1 $\rightarrow 2)$ - $\alpha$-D-mannopyranosyl-( $1 \rightarrow 6) \mid-\alpha$ - D-mannopyranosyl-(1 $\rightarrow 3)$-[ $\alpha$-D-mannopyranosyl-( $1 \rightarrow 2)$ - $\alpha$-D-mannopyranosyl-(1 $\rightarrow$ 6)]- $\alpha$-D-mannopyranoside (20)}

Octasaccharide 19 (604 mg, $0.20 \mathrm{mmol}$ ) was dissolved in a saturated solution of ammonia in $\mathrm{MeOH}(30 \mathrm{~mL})$. After $96 \mathrm{~h}$ at rt, the reaction mixture was concentrated, and the residue was purified by chromatography on Sephadex LH-20 $\left(\mathrm{H}_{2} \mathrm{O}\right)$ to afford $\mathbf{2 0}(173 \mathrm{mg}, 74.6 \%)$ as a foamy solid: ${ }^{1} \mathrm{H}$ NMR $\left(\mathrm{D}_{2} \mathrm{O}, 400 \mathrm{MHz}\right): \delta 5.01(\mathrm{~s}, 2$ $\mathrm{H}, 2 \mathrm{H}-1), 4.99$ (d, $\left.1 \mathrm{H}, J_{1,2} 0.6 \mathrm{~Hz}, \mathrm{H}-1\right), 4.91$ (d, $1 \mathrm{H}$, $\left.J_{1,2} 1.2 \mathrm{~Hz}, \mathrm{H}-1\right), 4.89$ (d, $\left.1 \mathrm{H}, J_{1,2} 1.2 \mathrm{~Hz}, \mathrm{H}-1\right), 4.86$ (d, $\left.1 \mathrm{H}, J_{1,2} 1.2 \mathrm{~Hz}, \mathrm{H}-1\right), 4.59$ (d, $\left.1 \mathrm{H}, J_{1,2} 0.8 \mathrm{~Hz}, \mathrm{H}-1\right)$, 
$3.28\left(\mathrm{~s}, 3 \mathrm{H}, \mathrm{OCH}_{3}\right) ;{ }^{13} \mathrm{C}$ NMR $\left(100 \mathrm{MHz}, \mathrm{D}_{2} \mathrm{O}\right): \delta$ 105.1, 105.1, 105.0, 104.9, 103.6, 100.6, 100.5 (7 C, 7 C1), $57.5\left(\mathrm{OCH}_{3}\right)$. MALDI-TOF MS Calcd for $\mathrm{C}_{43} \mathrm{H}_{74} \mathrm{O}_{36}: 1167.0$ [M]. Found: $1190.0\left[\mathrm{M}+\mathrm{Na}^{+}\right]$.

\section{Acknowledgements}

This work was supported by The Chinese Academy of Sciences (KZCX3-J-08) and by The National Natural Science Foundation of China (Projects 30070185 and 39970864).

\section{References}

1. Essentials of Glycobiology; Varki, A.; Cummings, R.; Esko, J.; Freeze, H.; Hart, G.; Marth, J., Eds.; Cold Spring Harbor Laboratory: Cold Spring Harbor, New York, 1999.

2. Dwek, R. A. Chem. Rev. 1996, 96, 683-720.

3. Grice, P.; Ley, S. V.; Pietruszka, J.; Priepke, H. W. M. Angew. Chem., Int. Ed. Engl. 1996, 35, 197-200.

4. Wyss, D. F.; Choi, J. S.; Li, J.; Knoppers, M. H.; Willis, K. J.; Arulanandam, A. R. N.; Smolyar, A; Reinherz, E. L.; Wagner, G. Science 1995, 269, 1273-1298.
5. (a) Ning, J.; Kong, F. Tetrahedron Lett. 1999, 13571360 ;

(b) Heng, L.; Ning, J.; Kong, F. Carbohydr. Res. 2001, 331, 431-438;

(c) Ning, J.; Heng, L.; Kong, F. Tetrahedron Lett. 2002 , 673-675.

6. (a) Zhang, J.; Kong, F. Tetrahedron: Asymmetry 2002, 13, 243-252;

(b) Zhu, Y.; Kong, F. Synlett 2001, 1217-1220;

(c) Zhu, Y.; Kong, F. Synlett 2000, 1783-1787;

(d) Du, Y.; Zhang, M.; Kong, F. Tetrahedron 2001, 57, 1757-1763;

(e) Zhu, Y.; Chen, L.; Kong, F. Chin. J. Chem. 2001, 19, 1289-1295.

7. (a) J. Ning; W. Zhang; Y. Yi; G. Yang; Z. Wu; J. Yi; F. Kong, Bioorg. Med. Chem., 2003, in press.;

(b) Ning, J.; Yi, Y.; Kong, F. Tetrahedron Lett. 2002, 5545-5549.

8. Zhang, J.; Kong, F. Tetrahedron Lett. 2003, 18391842.

9. Copeland, C.; Stick, R. V. Aust. J. Chem. 1978, 31, 1371.

10. Schmidt, R. R.; Kinzy, W. Adv. Carbohydr. Chem. Biochem. 1994, 50, 21-125.

11. Zhu, Y.; Kong, F. Synlett 2000, 1783-1787.

12. (a) Zhu, Y.; Kong, F. Synlett 2000, 663-667;

(b) Zhu, Y.; Kong, F. Carbohydr. Res. 2001, 332, 1-21. 